\title{
A magnetic source imaging camera
}

\author{
V. Dolgovskiy, ${ }^{1, a)}$ I. Fescenko, ${ }^{1,2}$ N. Sekiguchi, ${ }^{1,3}$ S. Colombo, ${ }^{1}$ V. Lebedev, ${ }^{1}$ J. Zhang,,${ }^{1,4}$ \\ and $A$. Weis \\ ${ }^{1}$ Physics Department, University of Fribourg, 1700 Fribourg, Switzerland \\ ${ }^{2}$ Institute of Atomic Physics and Spectroscopy, University of Latvia, 1050 Riga, Latvia \\ ${ }^{3}$ Department of Applied Physics, Tokyo University of Agriculture and Technology, Tokyo 184-8588, Japan \\ ${ }^{4}$ College of Science, Harbin Engineering University, 150001 Harbin, People's Republic of China
}

\begin{abstract}
We describe a magnetic source imaging camera (MSIC) allowing a direct dynamic visualization of the two-dimensional spatial distribution of the individual components $B_{x}(x, y), B_{y}(x, y)$ and $B_{z}(x, y)$ of a magnetic field. The field patterns allow - in principle - a reconstruction of the distribution of sources that produce the field $\vec{B}$ by inverse problem analysis. We compare experimentally recorded point-spread functions, i.e., field patterns produced by point-like magnetic dipoles of different orientations with anticipated field patterns. Currently, the MSIC can resolve fields of $\approx 10 \mathrm{pT}$ ( $1 \mathrm{~s}$ measurement time) range in a field of view up to $\sim 20 \times 20 \mathrm{~mm}^{2}$. The device has a large range of possible applications. As an example, we demonstrate the MSIC's use for recording the spatially resolved N'eel magnetorelaxation of blocked magnetic nanoparticles.
\end{abstract}

In recent years, optically pumped atomic magnetometers (AMs), also known as optical magnetometers, operated by laser light have achieved intrinsic magnetometric sensitivities in the $\mathrm{fT} / \sqrt{\mathrm{Hz}}$ range ${ }^{1}$ and sub-fT $/ \sqrt{\mathrm{Hz}}$ range $^{2}$ that are comparable to the performance of standard SQUIDmagnetometers. A comprehensive review of AM methods is given, e.g., in Ref. 3.

For mapping magnetic field distributions over extended regions of space, one may scan a single (scalar or vector component) magnetometer through the volume of interest, a very time-consuming method. Taue et al. ${ }^{4}$ have demonstrated $10 \mathrm{pT}$ magnetometric and mm spatial resolutions by scanning a single high-sensitivity AM through the field produced by an object. AMs have recently been deployed for eddy current imaging of electrically conductive materials yielding a sub-mm resolution. ${ }^{5,6}$ Very recently, a flux-guide based AM microscope has demonstrated a resolution of $250 \mu \mathrm{m}$ and a sensitivity of $23 \mathrm{pT} / \sqrt{\mathrm{Hz}}{ }^{7}$ However, such sequential measurements are speed-limited by the involved mechanical motion and can hence not be used for time- and space-resolved recordings or direct field visualization. A more efficient approach involves arrays of individual magnetometers, ${ }^{8-11}$ each containing an individual vapour cell and a photodetector. The spatial resolution in that case is determined by the number of sensors, the achieved signal/noise ratio, and source reconstruction algorithms.

In most AM-based field-mapping devices, the magnetometric information of interest is encoded into the intensity (or polarization) of atomic resonance radiation. Nonlinear magneto-optical effects (reviewed, e.g., in Ref. 12) form the basis for such an optical encoding. In this letter, we describe a magnetic source imaging camera (MSIC) that builds on this principle. We detect fluorescence from a single atomic vapour cell by a CCD camera, the camera pixels playing the role of individual photodetectors. The parallel recording and

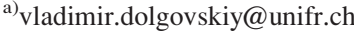

processing of all camera pixels can be interpreted in terms of an identical number of magnetometer signals, which make the MSIC a magnetometer with a high spatial resolution. Such a device has the potential for a wealth of practical applications, ranging from screening for magnetic material contaminations to biomedical imaging.

In the past, several experiments have reported the imaging of inhomogeneous distributions of spin-polarized atoms $^{13-15}$ or inhomogeneous magnetic fields ${ }^{16}$ using transmitted light detection. Recently, a research team at the University of Basel has demonstrated a method for imaging all vector components of a microwave field using CCD camera detection of bichromatic laser light traversing a MEMS cell. ${ }^{17,18}$ Ito et al. ${ }^{19}$ have demonstrated a transmitted light detection based multi-pixel magnetometer using a hybrid K$\mathrm{Rb}$ atomic vapour. Alternatively, one may use fluorescence detection for the field imaging, but reports on that approach are scarce. The seminal 1976 paper by Alzetta et al. ${ }^{20}$ was the first to illustrate (by photographic means) that the fluorescence intensity emitted by alkali vapour atoms depends on the atoms' degree of spin-polarization itself depending on the local magnetic field (dark vs. bright states). Photographic means were also used to realize an optical rf spectrum analyzer. $^{21,22}$ Asahi et al. ${ }^{23}$ reported images of one- and twodimensional field distributions using CCD detection of bichromatic dark states in $\mathrm{Na}$ vapour.

Fescenko and Weis ${ }^{24}$ have derived (and experimentally verified) algebraic equations that relate the spatial distribution of the fluorescence intensity emitted by a thin twodimensional layer of Cs atoms in a buffer gas to the orientation and magnitude of the field in that layer. The methods elaborated in that paper form the basis for inferring quantitative magnetic field values from the recorded fluorescence intensities. Compared to transmission detection, fluorescence detection suffers from the finite solid angle of fluorescence collection but has the advantage of avoiding perturbing interference effects encountered in transmission, i.e., forward 
scattering. Moreover, fluorescence recording detects the signal on an (ideally) black background, a definite advantage compared to transmission experiments in which the signal of interest may only be a small fraction of the total detected light intensity, thus imposing less severe constraints on the light intensity's stability and the camera's dynamic range.

The MSIC is based on imaging the fluorescence from a $\sim 20 \times 20 \times 0.3 \mathrm{~mm}^{3}$ layer of spin-polarized Cs atoms onto a CCD camera. The atoms are contained as a room-temperature vapour, together with a buffer gas mixture $(8 \mathrm{mbar} \mathrm{Ar}$ and 45 mbar $\mathrm{Ne}$ ) in a cubic Pyrex cell. A circularly polarized beam from an $894 \mathrm{~nm}$ extended-cavity diode laser (Toptica, model DL100), frequency-locked to the $4 \rightarrow 3$ hyperfine transition of the caesium $\mathrm{D}_{1}$ line, produces the layer of polarized atoms by optical pumping. Prior to entering the cell, the laser beam is prepared into a vertically oriented rectangular shape $\left(\sim 20 \times 0.3 \mathrm{~mm}^{2}\right)$ by means of a Gauss-to-top-hat beam shaper lens (TOPAG Lasertechnik, model GTH-5-250-4-IR) in combination with a cylindrical lens and a mask.

The device is located in a cylindrical two-layer mu-metal magnetic shield with open ends. The inner $130 \mathrm{~cm}$ long cylinder has a diameter of $26 \mathrm{~cm}$. Two mutually orthogonal pairs of rectangular coils and a solenoid along the shield axis are used to apply a well defined homogeneous magnetic field.

Figure 1 shows a schematic view (components not to scale) of the experimental arrangement. The laser-induced fluorescence from the irradiated Cs vapour layer is imaged onto a 14-bit $1384 \times 1032$ pixel CCD camera (Point Grey, model Grasshopper GS3-U3-15S5M-C) with a compact $50 \mathrm{~mm}$ fixed focal length lens (Edmund Optics, model VIS-NIR 67717) protected by an interference filter suppressing ambient light. The recorded LIF intensity variations cover $\sim 17 \%$ of the $2^{14}$ grey tones delivered by the camera. The image of the $\sim 20 \times 20 \mathrm{~mm}^{2}$ fluorescing source covers an area of $\sim 640 \times 640$ pixels that we bin into $160 \times 160$ groups of 16 pixels each. The size of each binned pixel group (BPG) thus represents an area of $130 \times 130 \mu \mathrm{m}^{2}$ in the fluorescence emitting plane. Binned frames are recorded at a rate of $4 \mathrm{fps}$ (frames per second), each frame being exposed for $210 \mathrm{~ms}$. We refer to a coordinate system with the origin located in the center of the fluorescence plane. We denote the coordinate axes by $\hat{k}$ (along the laser beam), $\hat{H}$ (horizontal), and $\hat{V}$ (vertical), respectively.

In order to understand the field reconstruction algorithm, we first recall the basics of bright/dark state spectroscopy: A homogeneous magnetic field $B_{k}$ applied along the laser beam direction stabilizes the atomic spin polarization, thus

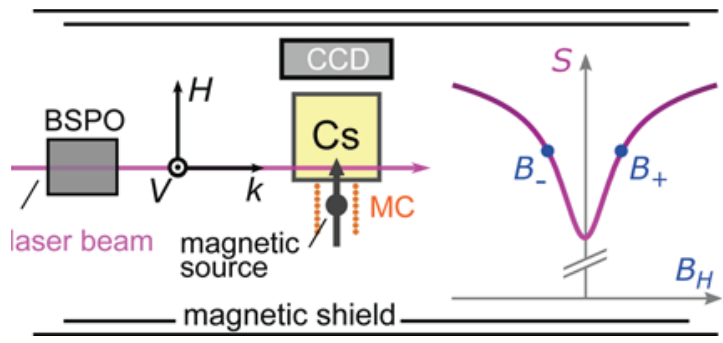

FIG. 1. Experimental apparatus (BSPO: beam-shaping and polarization optics; CCD: charge-coupled device; MC: magnetization coil for nanoparticles). Inset: Typical zero-field level crossing (Hanle) resonance of the fluorescence yield $S$. Choosing working points $B_{+}$or $B_{-}$at the half-height of the resonance yields linear responses $\pm \delta S$ to small changes $\delta B_{H}$. creating a dark state that yields a (homogeneous) lowintensity CCD image, while a transverse field $B_{V}$ or $B_{H}$ will depolarize the atoms and produce a bright image. The resonant variations of the intensity $S$ (defined as the number of detected fluorescence photons in a given time interval) that one observes when scanning either one of the three field components $B_{\text {scan }}=\{k, H, V\}$ are known as longitudinal $\left(B_{k^{-}}\right.$ scan) and transverse $\left(B_{H^{-}}\right.$or $\left.B_{V^{-s c a n}}\right)$ ground state Hanle resonances. Castagna and Weis have derived (and verified) explicit algebraic formulas for both variants of the Hanle effect. $^{25}$ The variations manifest themselves in $S\left(B_{\text {scan }}\right)$ plots as Lorentzian-shaped resonances ("zero-field level crossing resonances") centered at $B_{\text {scan }}=0$. An example for the dark resonance detected by one of the $\sim 10^{4} \mathrm{BPGs}$ in a $B_{H^{-}}$-scan is shown as the inset in Fig. 1. The same graph also illustrates the detection method: When fixing $B_{H}$ to one of the two "offset" values (solid blue dots) $B_{ \pm}= \pm \Delta B_{\mathrm{HW}}, \Delta B_{\mathrm{HW}}$ being the half-width of the Hanle resonance, any small additional component $\delta B_{H}$ will change the fluorescence level by a signed amount that is proportional to $\delta B_{H}$, provided that $\delta B_{H} \ll \Delta B_{\mathrm{HW}}$. By orienting the offset field along either one of the three coordinate directions, we can thus record selectively variations $\delta B_{k}, \delta B_{H}$, or $\delta B_{V}$.

Typical data recording proceeds as follows: Prior to doing actual source mapping experiments, we record a series of reference images $S_{\text {ref }}$ in order to account for the inhomogeneity and anisotropy of the offset field across the camera's field of view. We alternate the offset field from $B_{+}$to $B_{-}$, such that the signals of odd- and even-numbered frames represent $S_{\text {ref }}^{+}=S_{\text {ref }}\left(B_{+}\right)$and $S_{\text {ref }}^{-}=S_{\text {ref }}\left(B_{-}\right)$, respectively. We define-for each BPG-a normalized background as $S_{\text {bgd }}=\left(S_{\text {ref }}^{+}-S_{\text {ref }}^{-}\right) /\left(S_{\text {ref }}^{+}+S_{\text {ref }}^{-}\right)$that is independent of light intensity and atomic density variations. From the Hanle theory, ${ }^{25}$ one easily shows that $S_{\text {bgd }}=\delta B_{\text {scan }} / \Delta B_{\text {HW }}$ $+\mathcal{O}\left(\delta B_{\text {scan }}^{3} / \Delta B_{\mathrm{HW}}^{3}\right)$, where we have replaced the exemplary $\delta B_{H}$ by the more general field $\delta B_{\text {scan. Perfectly homogeneous }}$ offset fields $B_{ \pm}$would yield background images with a homogeneous grey tone. In reality, we find a smooth $\sim 0.5 \%$ variation of grey tones across the field of view.

We have tested the MSIC's performance by recording magnetic dipole point-spread functions (MD-PSF), i.e., fluorescence images produced by a point-like magnetic dipole, located $3 \mathrm{~mm}$ from the outer cell window on the camera's line of sight. The point dipoles were realized by three identical, but mutually orthogonal $5 \mathrm{~mm}$ diameter circular coils wound on the same support structure. This allowed us, at will, to produce a magnetic moment $\vec{\mu}_{j}$ oriented along any of the coordinate axes $\hat{k}, \hat{H}$, or $\hat{V}$. We power each coil sequentially and record signal images $S^{ \pm}=S\left(B_{ \pm}\right)$using the same alternating frame procedure as for the reference images. We finally define a background-subtracted normalized differential frame (BSNDF) image as

$$
\delta S_{i} \equiv\left(S^{+}-S^{-}\right) /\left(S^{+}+S^{-}\right)-S_{\mathrm{bgd}}=\delta B_{i} / \Delta B_{\mathrm{HW}},
$$

in which the individual BPG intensities depend in a linear manner on the local magnetic field component of interest, provided that $\delta B_{i} \ll \Delta B_{\mathrm{HW}}$, where $i=\{k, H, V\}$.

Since by an appropriate choice of the offset field direction, we can selectively detect each of the three field 
components, produced by each of the three principal magnetic moment orientations; there are 9 PSFs consisting of four distinct patterns (up to rotations and dark/bright inversions). The corresponding fluorescence patterns for $\Delta x=6.5 \mathrm{~mm}$ (see top of Fig. 2) are readily evaluated using the analytic expression for magnetic field components of a $5 \mathrm{~mm}$ diameter circular current loop. ${ }^{26}$ The same figure shows a comparison between the anticipated patterns (contour lines) and the corresponding experimentally recorded PSFs (density plots). For ease of comparison, both the experimental and theoretical pixel intensity distributions were mapped onto the interval $[0,1]$, the values 0 and 1 corresponding to the lowest and highest intensities in each of the 9 PSF images, respectively. The observed and modeled fluorescence patterns show an excellent agreement.

The comparison of theoretical and experimental PSFs represents a forward problem consisting in predicting (and verifying) intensity patterns from a known source. For practical applications, one has to solve the much more demanding inverse problem that consists in inferring the unknown distribution of magnetic sources from the observed fluorescence pattern. For this purpose, the experimentally recorded PSFs will serve as kernels in numerical deconvolution procedures. Work on source reconstruction is in progress.

In the final part, we discuss an application of the MSIC, viz., the spatially resolved recording of magnetorelaxation (MRX) of a small magnetic nanoparticle sample. Magnetic nanoparticles (MNPs), such as superparamagnetic iron oxide
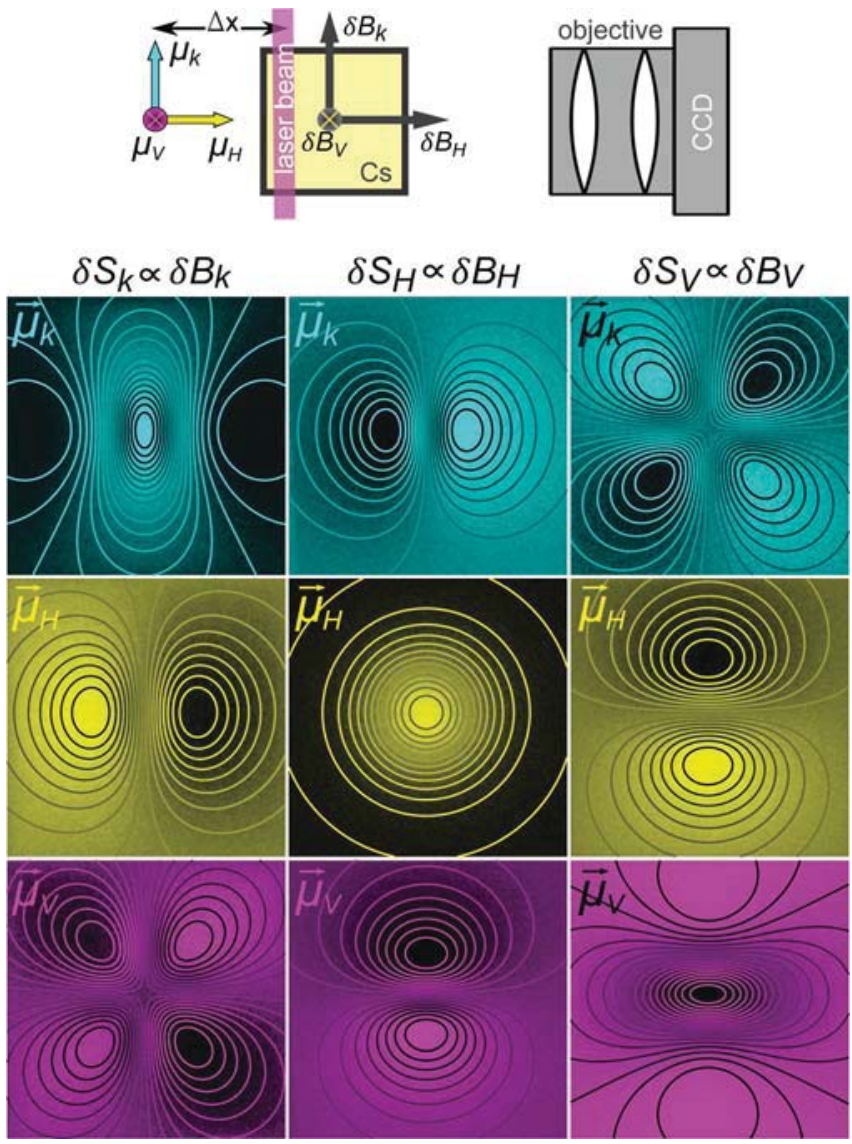

FIG. 2. Density plots representing measured MD-PSFs of individual field components produced by a point-dipole oriented along the $(\hat{k}, \hat{H}, \hat{V})$ axes, as shown in the top sketch. Contour lines represent theoretical predictions. nanoparticles, play a role of rapidly increasing importance in biomedical diagnostics and therapy. ${ }^{27}$ Therapeutic applications call for imaging modalities allowing the quantitative assessment of MNP distributions in/on specific biological entities, e.g., organs or tumors. The techniques of spatially resolved magnetorelaxation $(\mathrm{MRX})^{28}$ and Magnetic Particle Imaging (MPI) ${ }^{29}$ are promising recent approaches for imaging immobilized MNPs or MNPs suspended in body fluids, respectively. In a previous study, ${ }^{30}$ we have shown that atomic magnetometry (without spatial resolution) is a powerful method for high precision MRX studies. Here, we have placed a dried sample of $200 \mathrm{~nm}$ diameter $\mathrm{Fe}_{2} \mathrm{O}_{3}$ (maghemite) particles (fluidMAG/CF-D from Chemicell) sealed in a nylon tube container ( $3 \mathrm{~mm}$ long, $2 \mathrm{~mm}$ inner diameter) in front of the MSIC window; the sample thus replacing the magnetic dipole in the top graph of Fig. 2.

The sample is magnetized during $\sim 10 \mathrm{~s}$ by a $\sim 2.5 \mathrm{mT}$ DC magnetic field applied along the camera's line of sight ( $\hat{H}$-direction). After switching off the magnetizing field, we record the time evolution of the $\delta B_{H}$ field pattern produced by the relaxing MNPs' magnetic moment $\vec{\mu}_{H}$ (central pattern of Fig. 2). The inset of Fig. 3 shows typical BSNDF images $(0.5 \mathrm{~s}$ effective acquisition time) from such a relaxation time series. We average the resulting pixel intensity distribution over the circular region of interest (60 bin radius, centered at the intensity distribution's center of mass) marked by a dashed line in Fig. 3. These averaged values are then plotted (blue dots) as a function of time, yielding the MRX signal. The solid red line is a fit of the data with the function $\delta B_{H}(t)=B_{0} \ln (1+\tau / t)$ that is typical for a size-dispersed MNP sample. ${ }^{30}$ The fit residuals shown in the lower part of the figure illustrate the excellent agreement with the model function, thus proving the superparamagnetic character of the sample. The small deviation for the first few points is due to the fact that at early times $\delta B_{H}$ is so large that the linearization assumption $\delta B_{H} \ll \Delta B_{\text {HW }}$ discussed earlier is violated. We were able to detect MRX signals up to $\sim 10$ min following the sample's magnetization. A quantitative determination of the amount of immobilized MNPs can be performed using the detailed analysis method ${ }^{30}$ that we have developed recently.

The dynamic range of the method is limited by the Hanle linewidth that has a lowest achievable value $\Delta B_{\mathrm{HW}}$ of

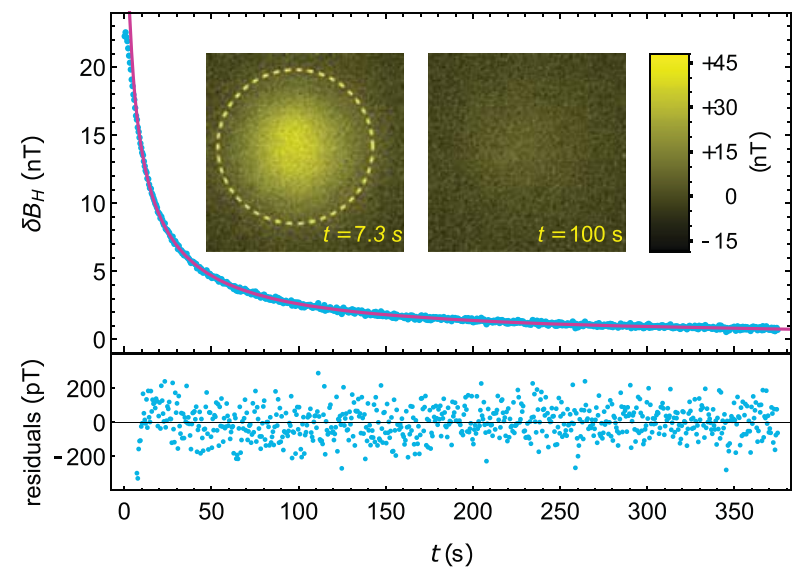

FIG. 3. Inset: Selected BSNDF images of the magnetorelaxation of an MNP sample. Data: MRX decay curve of averaged data from the region of interest, marked by dashed line, together with fitted time dependence $\left(B_{0}=10.2 \mathrm{nT} ; \tau=29 \mathrm{~s}\right)$. 
$\sim 10 \mathrm{nT}$ in our room-temperature cell. Since the data shown in Fig. 3 surpass that value, we broadened the resonance to $\approx 80 \mathrm{nT}$ by adding a small $B_{k}$ field component. The fit residuals' rms value of $\sim 100$ pT scales down to $\sim 13$ pT if a resonance with 10 , rather than $80 \mathrm{nT}$ half-width is used. We can thus claim a magnetometric sensitivity $\approx 10 \mathrm{pT}$ (for a $1 \mathrm{~s} \mathrm{ac}$ quisition time) when using information from all pixels in the $\sim 2 \mathrm{~cm}^{2}$-sized region of interest.

The spatial resolution $\delta x$ of the method is the precision with which the center of the pattern produced by a point-like dipole can be inferred from the MSIC image. One can show that $\delta x=\Delta x / \mathrm{SNR}$, where $\Delta x$ is the dipole-to-fluorescence plane distance (Fig. 2) and SNR the signal-to-noise ratio that depends on the source field strength.

In ongoing studies, we address small mismatches between the expected and observed PSF patterns, image blurring due to the diffusion of the Cs atoms as well as pattern deformations originating from atom-wall collisions. The presented method for visualizing magnetic fields may find applications in the localization and quantification of magnetic source distributions, such as, e.g., the detection of sentinel lymph nodes stained by MNPs. ${ }^{31}$

This work was partially funded by Swiss National Science Foundation Grant No. 200021_160128. I.F., N.S. and J.Z. acknowledge financial support from Pool de Recherche de l'Université de Fribourg, TOBITATE! Young Ambassador Program, and China Scholarship Council, respectively. We thank A. Petri-Fink and B. Michen for preparing the MNP sample.

${ }^{1}$ N. Castagna, G. Bison, G. Di Domenico, A. Hofer, P. Knowles, C. Macchione, H. Saudan, and A. Weis, Appl. Phys. B 96, 763 (2009).

${ }^{2}$ I. K. Kominis, T. W. Kornack, J. C. Allred, and M. V. Romalis, Nature 422, 596 (2003).

${ }^{3}$ D. Budker and M. Romalis, Nat. Phys. 3, 227 (2007).

${ }^{4}$ S. Taue, Y. Sugihara, T. Kobayashi, K. Ishikawa, and K. Kamada, Jpn. J. Appl. Phys., Part 1 50, 116604 (2011).
${ }^{5}$ C. Deans, L. Marmugi, S. Hussain, and F. Renzoni, Appl. Phys. Lett. 108, 103503 (2016)

${ }^{6}$ A. Wickenbrock, N. Leefer, J. W. Blanchard, and D. Budker, Appl. Phys. Lett. 108, 183507 (2016).

${ }^{7}$ Y. J. Kim and I. Savukov, Sci. Rep. 6, 24773 (2016).

${ }^{8}$ K. Sternickel and A. I. Braginski, Supercond. Sci. Technol. 19, S160 (2006).

${ }^{9}$ G. Bison, N. Castagna, A. Hofer, P. Knowles, J.-L. Schenker, M. Kasprzak, H. Saudan, and A. Weis, Appl. Phys. Lett. 95, 173701 (2009).

${ }^{10}$ G. Lembke, S. N. Erné, H. Nowak, B. Menhorn, A. Pasquarelli, and G. Bison, Biomed. Opt. Express 5, 876 (2014).

${ }^{11}$ P. Knowles, G. Bison, N. Castagna, A. Hofer, A. Mtchedlishvili, A. Pazgalev, and A. Weis, Nucl. Instrum. Methods Phys. Res., Sect. A 611, 306 (2009).

${ }^{12}$ D. Budker, W. Gawlik, D. F. Kimball, S. M. Rochester, V. V. Yashchuk, and A. Weis, Rev. Mod. Phys. 74, 1153 (2002).

${ }^{13}$ J. Skalla, G. Wäckerle, M. Mehring, and A. Pines, Phys. Lett. A 226, 69 (1997).

${ }^{14}$ K. Ishikawa, Y. Anraku, Y. Takahashi, and T. Yabuzaki, J. Opt. Soc. Am. B 16, 31 (1999).

${ }^{15}$ D. Giel, G. Hinz, D. Nettels, and A. Weis, Opt. Express 6, 251 (2000).

${ }^{16}$ E. E. Mikhailov, I. Novikova, M. D. Havey, and F. A. Narducci, Opt. Lett. 34, 3529 (2009).

${ }^{17}$ P. Böhi and P. Treutlein, Appl. Phys. Lett. 101, 181107 (2012).

${ }^{18}$ A. Horsley, G.-X. Du, and P. Treutlein, New J. Phys. 17, 112002 (2015).

${ }^{19}$ Y. Ito, D. Sato, K. Kamada, and T. Kobayashi, IEEE Trans. Magn. 50, 1-3 (2014).

${ }^{20}$ G. Alzetta, A. Gozzini, L. Moi, and G. Orriols, Il Nuovo Cimento B 36, 5 (1976).

${ }^{21}$ A. C. Tam and W. Happer, Appl. Phys. Lett. 30, 580 (1977).

${ }^{22}$ A. C. Tam, J. Appl. Phys. 50, 1171 (1979).

${ }^{23}$ H. Asahi, K. Motomura, K.-i. Harada, and M. Mitsunaga, Opt. Lett. 28, 1153 (2003).

${ }^{24}$ I. Fescenko and A. Weis, J. Phys. D: Appl. Phys. 47, 235001 (2014).

${ }^{25}$ N. Castagna and A. Weis, Phys. Rev. A 84, 053421 (2011).

${ }^{26}$ J. C. Simpson, J. E. Lane, C. D. Immer, and R. C. Youngquist, "Simple analytic expressions for the magnetic field of a circular current loop," Technical Report No. NASA/TM-2013-217919, 2001.

${ }^{27}$ S.-H. Huang and R.-S. Juang, J. Nanopart. Res. 13, 4411 (2011)

${ }^{28}$ R. Kötitz, T. Bunte, W. Weitschies, and L. Trahms, J. Appl. Phys. 81, 4317 (1997).

${ }^{29}$ B. Gleich and J. Weizenecker, Nature 435, 1214 (2005).

${ }^{30}$ V. Dolgovskiy, V. Lebedev, S. Colombo, A. Weis, B. Michen, L. Ackermann-Hirschi, and A. Petri-Fink, J. Magn. Magn. Mater. 379, 137 (2015).

${ }^{31}$ J.-L. Houpeau, M.-P. Chauvet, F. Guillemin, C. Bendavid-Athias, H. Charitansky, A. Kramar, and S. Giard, J. Surg. Oncol. 113, 501 (2016). 